\title{
NANO TECHNOLOGY: MOLECULAR SIMULATION
}

\author{
Guruprasad P Yennikoppa ${ }^{1}$, Ravi Katukam ${ }^{2}$ \\ ${ }^{1}$ Sr. Undergrad student, Indian Institute of Technology, Kharagpur, India \\ ${ }^{2}$ Manager, Cyient Limited, Hyderabad, India
}

\begin{abstract}
Laboratories throughout the world are trying to gain atomically precise control over the matter. This is because all materials are made up of atoms. Material behavior can be easily tracked by analyzing atomic behavior. Also studying at an atomic level help us in resolving problems which are due to atomic level phenomenon like crack propagation. Material model of atoms help in solving many industrial applications such as nanocutting of material, fracture and crack propagation, material deformation including corrosion ete.In this study a brief literature about molecular dynamics simulation is explained. Using this and the existing models an algorithm is framed for a general molecular dynamics simulation in 3-dimension. The same code with some modifications is used to study the behavior of molecules to explain nanocutting in materials and finding mechanical properties of material using molecular dynamics simulation.
\end{abstract}

Keywords: Nano technology, Molecular Simulation, Nano Cutting, Mechanical Properties $* * *$

\section{INTRODUCTION}

An extensive theoretical analysis is required to understand the properties and mechanism of nano-scale processes. Nano-scale processing operates at length scale of only a few atomic layers at the surface. At such a small governing length scale, the continuum representation of the problem becomes questionable. Hence we need a Molecular Dynamics (MD) model that can obtain the properties of molecules at nano-scale using trajectories of atoms.

Improved technologies have found a way in development of micro- and Nano-components in micro-electro-mechanical systems (MEMS) and micro-opto-electromechanical systems (MOEMS). The micro- and Nano-structural elements used in them are designed to withstand the complex stress state with minimal probability of failure. Also special processes are used to avoid defects while manufacturing structures using these materials. The ideal materials produced with less or few defects and a strength equals to their theoretical value are going to be typical with the exception for Nanocomponents in MEMS and MOEMS. Therefore an examination of strength as well as deformation of defect free materials is needed to analyze Nanocomponents.

In general Nano-indentation test with acutely small loads (usually in nano-Newtons) is used to find the mechanical properties of Nano-materials. The application of usual tensile test (used to find properties at macro level) is difficult or in way impossible due to short length and time scales. Some other significant factors that affect tensile testing of Nano-materials are highly expensive experimental set-up due to complexity of testing procedure. Also there is no efficient procedure to manufacture testing specimen with no or few defects. An alternative approach is MD simulations. Although fracture strength highly depends on the defect density, the strength of ideal materials also defends on the way it deforms and fail. In the following investigation, Molecular Dynamics Simulation is modeled in general for a 3-dimensional system. The developed MD model is modified for the following applications, first is Nano-cutting of copper by Diamond in 2-D and second is the tensile testing of 3-D model using Molecular Dynamics. Analysis performed and the conclusion of the work is made.

\subsection{Molecular Dynamics}

Molecular Dynamics (MD) is the solution of classical equations of motions for atoms and molecules to obtain the time evaluation of a system. It is applied to many particle systems since a general analytical solution is not possible. Hence it is computationally evaluated using numerical methods. Molecular Dynamics is used extensively as virtual experiment although it is not so accurate due to its affordability and ease of computing methods.

As we know any material is a collection of discrete atoms. In molecular dynamics simulation the idea of discrete atoms is used. The atoms interact by exerting forces on each other (internal forces) based on the interaction model developed. Thus following are the crucial ingredients of molecular dynamics simulation

- A potential model that describes the force field between atoms. It is usually called inter atomic potential U(r).

- Numerical integrator that is used to solve equations of motion.

- Initial and Boundary conditions.

- Finally educing the required data from the available atomic trajectory information. 


\subsection{Classical Mechanics: Newton's Equation of}

\section{Motion}

The real world problems can easily be portrayed by classical mechanics. if things are moving slow enough and are small enough that we need not consider relativity and things move fast enough and large enough that we don't have to worry about the quantum effects. From this we can understand that application of classical mechanics has both lower and upper boundary. Let's review the Newton's laws

First law: An object in a state of rest or uncelebrated motion will remain at the same state unless an external unbalanced force is applied to it. This is also called as Law of Inertia.

Second law: Net external force applied on an object is directly proportional to change in linear momentum of the object. Mathematically it can be described as

$F=m a \quad O R \quad F \propto \Delta(m u)$

$F=m \ddot{r} \quad$ OR $F \propto \Delta(m \ddot{r})$

Here, $F$ is force, $m$ is mass of the object and $a$ is the acceleration.

Third law: Every action has an equal and opposite reaction

Equations of motion for classical particles be it an atom or earths are obtained from Newton's Second law of motion. Also the same set of equations can be obtained using Lagrangian function and assuming that the force field between atoms is conservative, which is anyway true. The Lagrangian is defined as the difference between energy due to motion ( Kinetic) and potential energy.

$L(r, \dot{r})=T(\dot{r})-U(r)$

$F=-\frac{\partial U}{\partial r}$

Differentiating equation (3) with respect to time, position and velocity and using equation (4) we obtain equation (2) i.e.

$F=m \ddot{r}$

The reformulation of Newton's equation of motion using equation (4) is necessary as we use the potential energy function instead of force in molecular dynamics.

Equation (2) is a system of nonlinear ordinary differential equations with order two. In order to solve above system of equations we need two initial conditions per particle per dimension.

$r\left(t=t_{0}\right)=r_{0} \quad$ and $\dot{r}\left(t=t_{0}\right)=\dot{r}_{0}$
That is we need to know the position and velocity of each particle in each dimension at some instant of time. We generally solve Molecular Dynamics for $\mathrm{N}$ molecules, we have $3 \mathrm{~N}$ second order ordinary differential equations and hence initial conditions are needed to solve them are twice the number of equations. The computational space and time available decides upper limit for $\mathrm{N}$.

\subsubsection{Intermolecular Potentials}

In order to solve equation (2), we need potential energy function, U. Potential energy function depends on the type of molecules under analysis. Using subatomic theory of chemistry and physics $U$ can be derived for different types of molecules.

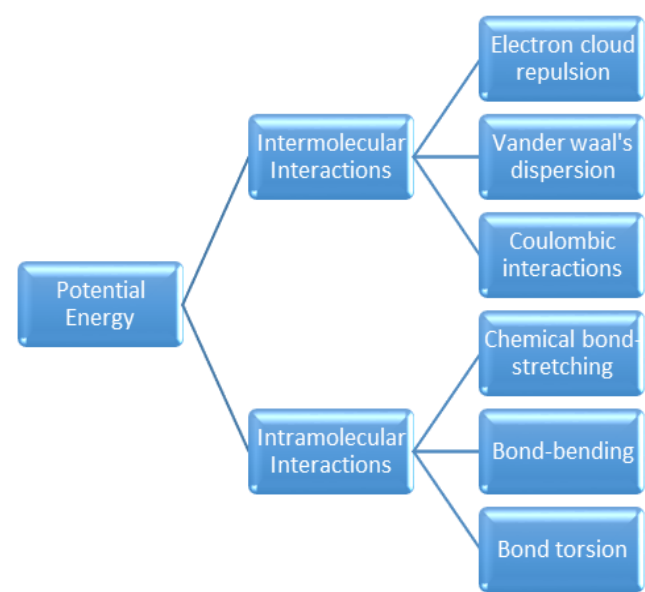

Fig 1 Potential Energy distribution

When molecules have degrees of freedom that can't be neglected, we need to consider intra-molecular interactions while calculating potential energy. Vibration and rotation about a chemical bond are included in internal degrees of freedom. In our study we only consider molecules with negligible degrees of freedom. Apart from this potential energy can be classified into pair-wise potentials and manybody potentials. Pair-wise potentials are calculated only using the possible interactions between two molecules. In our study we will restrict ourselves on considering only pairwise potentials only. Let's have a look at the different types of potential available in the literature

All the potential energy functions depend on the interatomic distance $r_{i j}$ which is defined as the magnitude of distance between centre of atoms $i$ and $j$.

$$
r_{i j}=\left|r_{i}-r_{j}\right|=\sqrt{\left(r_{x, i}-r_{x, j}\right)^{2}+\left(r_{y, i}-r_{y, j}\right)^{2}+\left(r_{z, i}-r_{z, j}\right)^{2}}
$$

Selection of proper potential energy function will highly impact the whole analysis. So choose the model wisely within the constraints of computational space and time available. In our study we have chosen Morse potential function as it suitable for metals. We have also done a simple gomparison with Lennard-Jones potential. It has been found that Embedded Atom Method (EAM) will give better accuracy than the pair-wise potentials under consideration. 


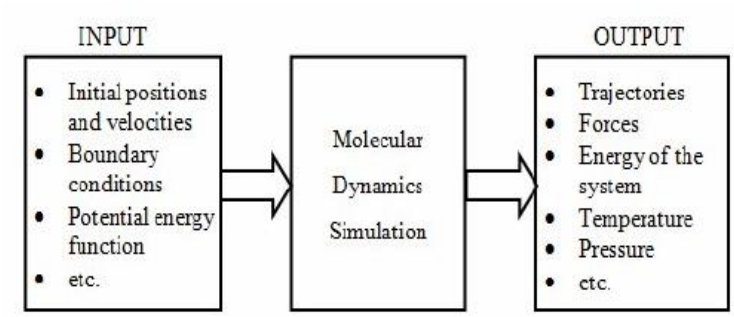

Fig-1: General Molecular Dynamics Model

Table 1 Potential Energy Functions

\begin{tabular}{|c|c|c|}
\hline Nan & Iodel & $\begin{array}{l}\text { Appli } \\
\text { catio } \\
\text { n }\end{array}$ \\
\hline $\begin{array}{l}\text { d- } \\
\text { Jones } \\
\text { Potenti } \\
\text { al }\end{array}$ & $\begin{array}{l}U_{L_{J}}=4 \varepsilon\left[\left(\frac{\sigma}{r}\right)^{12}-\left(\frac{\sigma}{r}\right)^{6}\right] \\
\sigma \text { and } \varepsilon \text { are constants which depend on } \\
\text { physical properties of the material }\end{array}$ & $\begin{array}{l}\text { Mostl } \\
\mathrm{y} \\
\text { suitab } \\
\text { le for } \\
\text { rare } \\
\text { gases }\end{array}$ \\
\hline $\begin{array}{l}\text { Morse } \\
\text { Potenti } \\
\text { al }\end{array}$ & $\begin{array}{l}U_{M P}=D\left\{\exp \left[-2 \alpha\left(r-r_{e}\right]-2 \exp \left[-\alpha\left(r-r_{e}\right]\right\}(7)\right.\right. \\
\mathrm{r} \text { and } \mathrm{r}_{\mathrm{e}} \text { are instantaneous and } \\
\text { equilibrium distance between two atoms } \\
\text { respectively, } \boldsymbol{\alpha} \text { and } \mathrm{D} \text { are constants } \\
\text { determined on the basis of physical } \\
\text { properties of the material. }\end{array}$ & $\begin{array}{l}\text { Mostl } \\
\text { y } \\
\text { suitab } \\
\text { le for } \\
\text { metal } \\
\text { s }\end{array}$ \\
\hline $\begin{array}{l}\text { Potenti } \\
\text { al }\end{array}$ & $\begin{array}{l}\text { A and } r_{0} \text { are constants dependent on the } \\
\text { material }\end{array}$ & $\begin{array}{l}\text { Mostl } \\
\text { y } \\
\text { suitab } \\
\text { le for } \\
\text { ceram } \\
\text { ics }\end{array}$ \\
\hline $\begin{array}{l}\text { Embed } \\
\text { ded } \\
\text { Atom } \\
\text { Metho } \\
\text { d } \\
\text { (EAM) } \\
\text { Potenti } \\
\text { al }\end{array}$ & $\begin{array}{l}E_{t o t}=\sum_{i} G_{i}\left(\rho_{h, i}\right)+\frac{1}{2} \sum_{i, j} U_{i j}\left(r_{i j}\right) \\
\rho_{\mathrm{h}, \mathrm{i}} \text { is the total electron density at atom } \mathrm{i} \\
\text { due to the rest of atoms in the system } \\
\mathrm{G}_{\mathrm{i}} \text { is the embedding energy for placing } \\
\text { an atom into theelectron density } \\
\mathrm{U}_{\mathrm{ij}} \text { is the short range pair interaction } \\
\text { representing the coreRepulsion } \\
\mathrm{r}_{\mathrm{ij}} \text { is the separation between atoms }\end{array}$ & $\begin{array}{l}\text { Mostl } \\
\text { y } \\
\text { suitab } \\
\text { le } \\
\text { for a } \\
\text { wide } \\
\text { range } \\
\text { of } \\
\text { metal } \\
\text { s }\end{array}$ \\
\hline
\end{tabular}
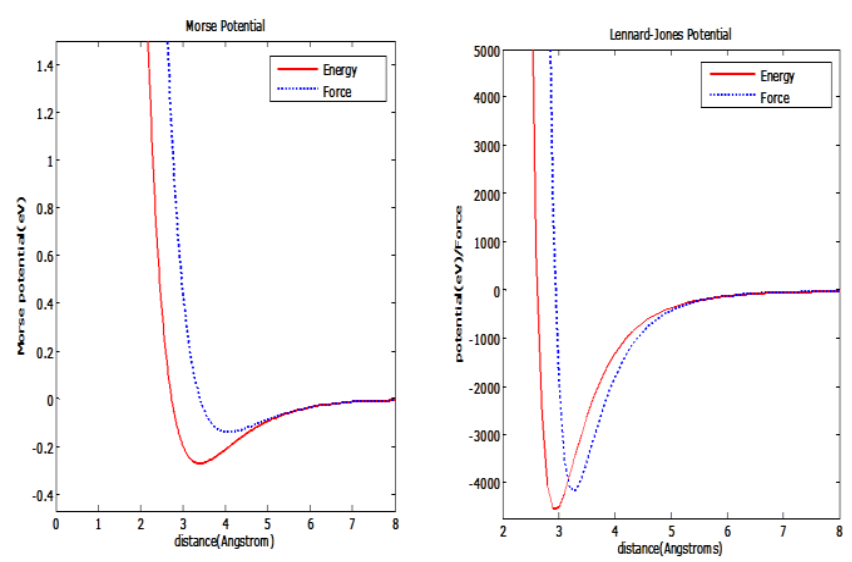

Fig 2 Lennar - Jones \& Lennar - Morse potential energy and force for Aluminium
Using equation (4) we can write the force generated in Lennard-Jones potential model and Morse potential model as follows

$$
F_{L J}=\frac{24 \varepsilon}{r_{i j}}\left[2\left(\frac{\sigma}{r_{i j}}\right)^{12}-\left(\frac{\sigma}{r_{i j}}\right)^{6}\right]
$$

\subsubsection{Time Integration Algorithm to Solve Equation}

\section{of Motion}

Some of the numerical integrator techniques that have been developed for solving equations of motion are the Verlet algorithm, the predictor-corrector algorithm and the Beeman's algorithm. The above integration techniques are developed by approximating thepositions, velocities and accelerations using a Taylor series expansion:

$r(t+\Delta t)=r(t)+\dot{r}(t) \Delta t+\frac{1}{2} r(t) \Delta t^{2}+\ldots$

$v(t+\Delta t)=\dot{r}(t)+\dot{r}(t) \Delta t+\frac{1}{2} \frac{r n}{r}(t) \Delta t^{2}+\ldots$

$a(t+\Delta t)=\stackrel{n}{r}(t)+\stackrel{m}{r}(t) \Delta t+\frac{1_{\text {mit }}}{2}(t) \Delta t^{2}+\ldots$

Where $\Delta \mathrm{t}$ is a finite time step, $\mathrm{r}$ is the position, $\mathrm{v}$ and $\stackrel{\vec{r}}{\text { are }}$ the velocity, a and $\stackrel{\ddot{r}}{ }$ are the acceleration and ${ }^{\ddot{r}}$ are the third and $\stackrel{r}{r}$ the fourth derivative of position with time.

\section{The Basic Verlet Algorithm}

Using this method, forward position can be predicted as;

$$
r(t+\Delta t)=r(t)+\dot{r}(t) \Delta t+\frac{1}{2} r(t) \Delta t^{2}+\ldots
$$

Similarly backward position;

$$
r(t-\Delta t)=r(t)-\dot{r}(t) \Delta t+\frac{1}{2} \vec{r}(t) \Delta t^{2}-\ldots
$$

From equations (13) and (14), we have

$$
r(t+\Delta t)=2 r(t)-r(t-\Delta t)+\ddot{r}(t) \Delta t^{2}
$$

Although this method doesn't explicitly use velocity vector for calculating positions it is not quite accurate. But also storage requirements are moderate and method of implementing is easier.

\section{The Verlet Leapfrog Algorithm}

In this method, average values halfway between position steps are used to find velocities. The equations are as follows; 
$r(t+\Delta t)=r(t)+\dot{r}\left(t+\frac{1}{2} \Delta t\right) \Delta t$

$v\left(t+\frac{1}{2} \Delta t\right)=\dot{r}\left(t-\frac{1}{2} \Delta t\right)+\vec{r}(t) \Delta t$

As the incremental time step for both position and velocity are different, both cannot be calculated simultaneously.

\section{The Velocity Verlet Algorithm}

Using equations deduced from equations (12) and (15), and ignoring infinitesimals, this algorithm calculates new positions, velocities and accelerations using their values at time, $\mathrm{t}$.

$r(t+\Delta t)=r(t)+\dot{r}(t) \Delta t+\frac{1}{2} \vec{r}(t) \Delta t^{2}$

$v(t+\Delta t)=\dot{r}(t)+\frac{1}{2}[\ddot{r}(t)+\ddot{r}(t+\Delta t)] \Delta t$

The velocity Verlet algorithm requires low memory.

\section{The Predictor-Corrector Algorithm}

Forward velocities in time are predicted and the forces calculated, and then the corrected forms of the velocities are later calculated. Combining the $r(t+\Delta t)$ and $a(t+\Delta t)$ in equation (12), the position can be written as:

$r(t+\Delta t)=r(t)+\dot{r}(t) \Delta t+\frac{2}{3} \vec{r}(t) \Delta t^{2}-\frac{1}{6} \vec{r}(t-\Delta t) \Delta t^{2}+0\left(\Delta t^{4}\right)$

The velocities at time $\mathrm{t}=\mathrm{t}+\Delta \mathrm{t}$ are then calculated from the positions. Combining the $\mathrm{r}(\mathrm{t}+\Delta \mathrm{t})$ and $\mathrm{a}(\mathrm{t}+\Delta \mathrm{t})$ in equation (20), the position can be expressed as:

(Predicted)

$v(t+\Delta t)=\dot{r}(t)+\frac{3}{2} \vec{r}(t) \Delta t-\frac{1}{2} \ddot{r}(t-\Delta t) \Delta t$

The acceleration at $\mathrm{t}=\mathrm{t}+\Delta \mathrm{t}$ are calculated from the positions and the predicted velocities.

(Corrected)

$v(t+\Delta t)=\dot{r}(t)+\frac{1}{3} \ddot{r}(t+\Delta t) \Delta t+\frac{5}{6} \tilde{r}(t) \Delta t-\frac{1}{6} \ddot{r}(t-\Delta t) \Delta t$

This algorithm can be called a self-checking one as it runs a comparison between the predicted and corrected values.

An appropriate numerical integration technique is chosen according to:

- $\quad$ Better accuracy - the result produced are close to exact solution Low memory requirement - a great concern
- Good efficiency - it should be fast enough and computationally efficient

- $\quad$ Time Step - it should permit large time step $\Delta \mathrm{t}$ for the integration

- $\quad$ Easy to implement - helps save time

Other integration algorithms like Euler methods or classical Fourth-Order Runge- Kutta method are not preferred due to high memory requirement and low efficiency.In our study we have chosen Gear Predictor Corrector Method due to high accuracy and efficiency, Velocity Verlet algorithm for its accuracy and low memory requirement. Now let's take look at both the algorithm in brief:

\section{Gear Predictor Corrector Method (GPC)}

A GPC method of order five is developed using the Taylor series expansion upto fifth derivative of the unknown variable (here it is position of atoms). Let us define a vector consisting of positions and its derivative pre-multiplied by Taylor series coefficients as

$$
R=\left[\begin{array}{llllll}
r & \Delta t \frac{d r}{d t} & \frac{\Delta t^{2} d^{2} r}{2} \frac{\Delta t^{3}}{d t^{2}} & \frac{d^{3} r}{6} \frac{\Delta t^{4}}{d t^{3}} & \frac{d^{4} r}{24} \frac{\Delta t^{5}}{d t^{4}} & \frac{d^{5} r}{120} \frac{d t^{5}}{t^{5}}
\end{array}\right]^{T}
$$

A new set of values for position and its derivative are predicted using the initial or previous values by Taylor series which in matrix form can be expressed as

$$
R^{p}=\left[\begin{array}{cccccc}
1 & 1 & 1 & 1 & 1 & 1 \\
0 & 1 & 2 & 3 & 4 & 5 \\
0 & 0 & 1 & 3 & 6 & 10 \\
0 & 0 & 0 & 1 & 4 & 10 \\
0 & 0 & 0 & 0 & 1 & 5 \\
0 & 0 & 0 & 0 & 0 & 1
\end{array}\right] R^{0}
$$

Note that the matrix in equation (26) is Pascal triangle. Now the forces are calculated using the potential model that we have considered. A new set of corrected values for position are calculated using the earlier predicted values and the force calculated using potential model.

$$
R^{c}=R^{p}+c\left[\frac{\Delta t^{2} F}{2}--\frac{\Delta t^{2} d^{2} r^{p}}{2} \frac{d t^{2}}{d t^{2}}\right]
$$

The vector $\mathrm{c}$ is simply a vector of constant corrector coefficient which is chosen in order to minimize the error and maximize the stability of system of equations.

For a second order ODE with fifth order GPC method where the forces are strictly a function of position (not velocity) the corrector coefficients are

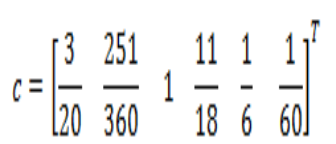


If the velocity does appear explicitly in the ODE (i.e. the force is a function of velocity), then the factor of $3 / 20$ should be replaced by a factor of $3 / 16$.

\section{Velocity Verlet Algorithm}

In this algorithm velocity and position are not updated at same time slices. The algorithm reads,

$r(t+\Delta t)=r(t)+v(t) \Delta t+\frac{1}{2} a(t) \Delta t^{2}$

$v\left(t+\frac{\Delta t}{2}\right)=v(t)+\frac{1}{2} a(t) \Delta t$

$a(t+\Delta t)=-\frac{1}{m} \frac{d V(r(t+\Delta t))}{d r(t+\Delta t)}$

$v(t+\Delta t)=v\left(t+\frac{\Delta t}{2}\right)+\frac{1}{2} a(t) \Delta t$

Although the accuracy of the algorithm is lower (especially in calculating velocity) than the higher order integrators due to its ease of implementation and low memory requirement it is usually preferred. Also speed of the simulation is greater in this case.

\section{Initial Conditions}

To solve a second order ODE we need two initial conditions in each dimension for a single particle. In this case they are position and velocity of atoms. We can begin the simulation in any configuration as initial conditions do not affect the equilibrium position unless there are local minima in Gibbs free energy. But in Molecular Dynamics simulation we will use standard initial conditions.

For the simulation of metals we will start the system with perfect simple cubic (SC) or face-centered-cubic (FCC) lattice. This is used for two reasons.

It is well elucidated and replicable configuration.

Overlapping of atoms is avoided.

If atoms overlap the system will "blow up" in few intial time steps due to $\mathrm{g}=$ high repulsive forces generated by overlapping atoms. Velocity of each particle at starting time is randomly initialized and then enforced with the following stipulations.

\section{Translational momentum must be conserved.}

Kinetic energy must be related to thermodynamic equipartition theorem,

$$
\frac{1}{2} \sum_{i=1}^{N} m_{i} \sum_{\alpha} v_{\alpha=x, y z}^{2}=\frac{3}{2} N k_{b} T
$$

Velocity distribution should follow Maxwell-Boltzmann distribution (optional).

\section{Boundary Conditions}

As we already have two initial conditions to solve the equations of motion, then what is the need of boundary conditions? Unlike other boundary conditions these periodic boundary conditions here are not used to solve the equations but rather are used to make the system infinite for simulation. Let consider a cubic system by imposing periodic boundary conditions in all three directions we can make the simulation system infinite in 3-dimensional space Hence by using Periodic Boundary Conditions (PBC) we can make the simulation to be periodic. These are exhibited in following ways

In the trajectories of particles. In the "minimum image convention"

Periodic boundary conditions are applied at the end of each time step to calculate the new positions of atoms inside the simulation volume. Conservation of energy in the simulation volume is ensured by the "minimum image convention", this ensures the energy of particle leaving and entering the simulation volume should be same. Because our simulation volume gets repeated periodically, the nearest atom to a atom say i, may not be the one next to it inside the simulation volume but may be lying near to the simulation volume.

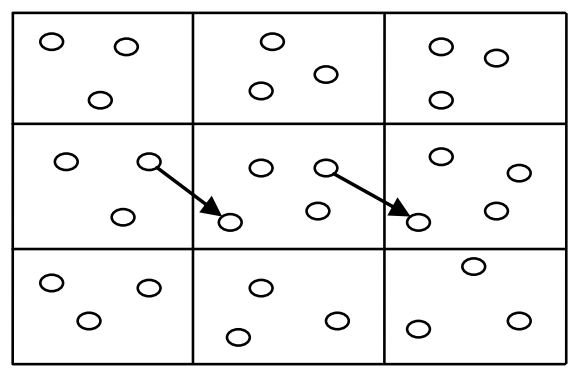

Fig 4 Periodic Boundary Conditions. When a molecule leaves the simulation volume, an image of the molecule enters the simulation volume

\section{Force Evaluation and Neighbor Lists}

In order to increase the efficiency of computing we introduce the idea of neighbor list and force evaluation using these neighbor lists. Neighbor list for an atom consists of nearby atoms placed within the cut-off distance rcut .For molecules separated by a distance greater than rcut or grater, we assume a mean-field with constant energy. Thus potential energy is a sum of short-range intermolecular forces and long-range approximation. For Lennard-Jones potential we have

$$
U=U_{S R}+U_{L R}=\frac{1}{2} \sum_{i=1}^{N} \sum_{\substack{j=1 \\ j \neq i \\ r_{i j} \leq r_{\text {cut }}}}^{N} U_{L J}\left(r_{i j}\right)+U_{L R}
$$




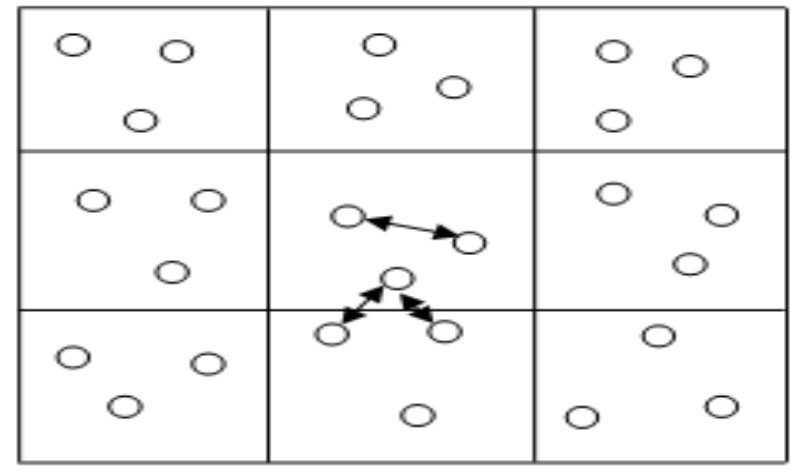

Fig 5 Minimum Image Convention. The separation of the pairs is defined by minimum separation between atom $i$ and any image of atom $\mathrm{j}$

$U_{L R}=\frac{1 N^{2}}{2} \frac{2 \pi}{V} \int_{0}^{2 \pi} \int_{0}^{\infty} \int_{r_{\text {cut }}}^{\infty} U_{L J}(r) \sin \theta r^{2} d r d \theta d \varphi=\frac{1 N^{2}}{2}\left[\frac{16 \pi \varepsilon}{9}\left(\frac{\sigma^{12}}{r_{\text {cut }}^{9}}-3 \frac{\sigma^{6}}{r_{\text {cut }}^{3}}\right)\right]$

Neighbor lists are used efficiently using a matrix with $\mathrm{m}$ rows and 2 columns, where $\mathrm{m}$ is the number of pairs of atoms possible within the cut-off distance. Both the columns give us the identification number for atoms under consideration for evaluating forces. This will increase our efficiency by reducing computational time and space.

\section{Property Evaluation}

Using the data available from the trajectories of atoms/molecules under Molecular Dynamics Simulation we can extract the output we need using suitable formulas such as,Potential energy from the potential energy model that we have chosenKinetic energy is given by sum of kinetic energies of individual molecule

$K E=\frac{1}{2} \sum_{i=1}^{N} m_{i} \sum_{\alpha=x, y, z} v_{\alpha}^{2}$

Temperature of the system can be obtained using thermodynamic equipartition theorem

$K E=\frac{1}{2} \sum_{i=1}^{N} m_{i} \sum_{\alpha=x, y z} v_{\alpha}^{2}=\frac{3}{2} N k_{b} T$

Displacement can be obtained by using initial and final positions.

\section{Molecular Dynamics Model in 3-D}

A general Molecular Dynamics Algorithm is developed for NVE ensemble, where $\mathrm{N}$ is number of molecules, $\mathrm{V}$ is volume of the system under simulation, $\mathrm{E}$ is total energy of the system. All three quantities are conserved for the system under simulation. A MATLAB code is written using the developed algorithm. The system is verified for NVE ensemble. Displacements of the molecules are obtained in order to check the trajectories of the molecules using which we evaluate the material properties. The following material properties are listed and calculated during the simulation
Table2 Material properties evaluated after Production step for aluminium metal

\begin{tabular}{|l|l|l|l|}
\hline $\begin{array}{l}\text { Property } \\
\text { name }\end{array}$ & $\begin{array}{l}\text { Instant } \\
\text { value }\end{array}$ & $\begin{array}{l}\text { Average } \\
\text { value }\end{array}$ & $\begin{array}{l}\text { Standard } \\
\text { Deviation }\end{array}$ \\
\hline Kinetic & $1.222047 \mathrm{e}+$ & $1.379896 \mathrm{e}+$ & $0.000000 \mathrm{e}+$ \\
Energy(aJ) & 00 & 00 & 00 \\
\hline Potential & $7.158807 \mathrm{e}+$ & $7.139253 \mathrm{e}+$ & $0.000000 \mathrm{e}+$ \\
Energy(aJ) & 01 & 01 & 00 \\
\hline Total & $7.281011 \mathrm{e}+$ & $7.277242 \mathrm{e}+$ & $0.000000 \mathrm{e}+$ \\
Energy(aJ) & 01 & 01 & 00 \\
\hline Temperature( & $4.720629 \mathrm{e}+$ & $5.330380 \mathrm{e}+$ & $0.000000 \mathrm{e}+$ \\
K) & 02 & 02 & 00 \\
\hline X-momentum & $-8.549174 \mathrm{e}-$ & $-1.830681 \mathrm{e}-$ & $0.000000 \mathrm{e}+$ \\
& 15 & 13 & 00 \\
\hline Y-momentum & $9.442951 \mathrm{e}-$ & $7.826464 \mathrm{e}-$ & $2.797582 \mathrm{e}-$ \\
& 14 & 14 & 07 \\
\hline Z-momentum & $7.849696 \mathrm{e}-$ & $-1.012574 \mathrm{e}-$ & $0.000000 \mathrm{e}+$ \\
& 14 & 14 & 00 \\
\hline
\end{tabular}

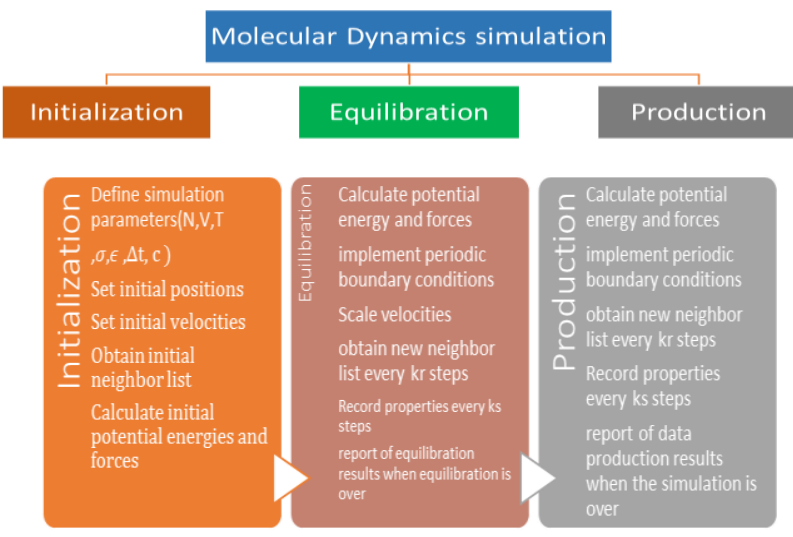

Fig 6: Algorithm overview

From figure 7, the total energy of the system is conserved throughout the simulation; similarly the number of atoms and the volume are also conserved. Hence the developed Molecular Dynamics Simulation is NVE ensemble. The atomic displacements of aluminium are evident as only vibration is involved in atoms of solid material. Also instantaneous properties, average and standard deviation of material properties are evaluated

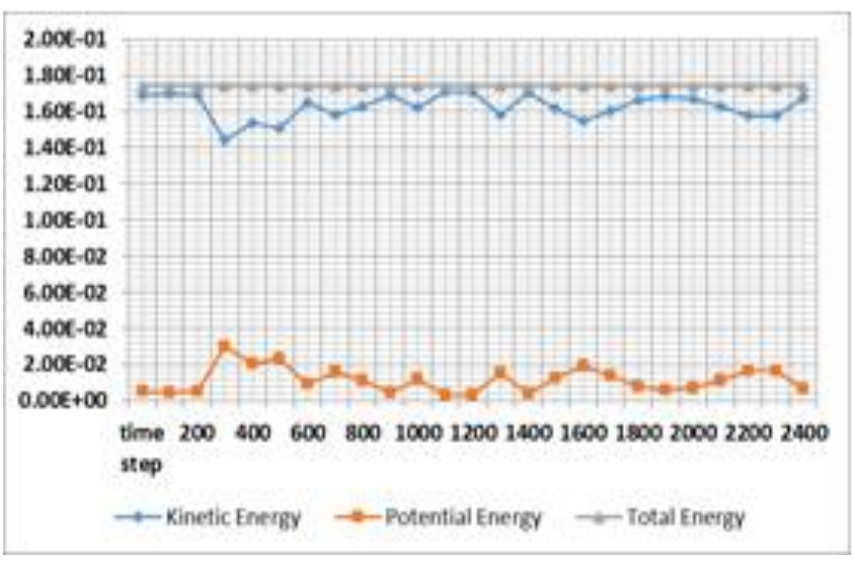

Fig 3 Verification of NVE ensemble 


\section{NANO SCALE CUTTING}

Chandrasekaran [8] investigated a method termed the Length Restricted Molecular Dynamics (LRMD) simulation to reduce the computational time and at the same time reduce the memory requirements significantly. Komanduri [9] reported MD simulation studies of machining with large negative rake angle tools to simulate grinding and compared the simulation results with the experimental results resulting in good agreement. Komanduri [9] also investigated the effect of tool geometry with tools of different edge radii relative to the depth of cut in nano metre. Lin and Huang [10] uses a method that combines molecular dynamics with finite element deformation model (MDFM) to calculate the stress and strain of single crystal copper that occur during nano-scale orthogonal cutting. They find that it has only minor influence for the different cutting speeds on the distribution of the strain and stress at the contact area between the cutting chip and rigid tool during steady cutting state. From the above literature, most of the literatures used in the nanocutting simulations use a rigid body tool to cut material in a vacuum environment. Most of the literatures have rarely focused on temperature fields created by cutting. By considering the tool as a rigid body, it has been unable to analyze the temperature change and wear of tool. For these reasons, this study constructed a molecular dynamics nanocutting simulation that considered the heat transfer of Elastic body tool to study the cutting conditions for single crystal copper material under different cutting parameters and to evaluate the temperature of the work piece and the tool, stress distribution, and machined surface quality during cutting.

\subsection{Nanocutting Simulation Model}

For our study of nanocutting using molecular dynamics simulation single crystal copper is the workpiece material (simulation was also performed on aluminium workpiece). A rigid body single crystal diamond is chosen as cutting tool. In this study cutting tool is assumed to be nondeformable (hence the diamond tool, the hardest material). The boundary conditions of nano-scale cutting simulation consists of four layer of atoms to left side of workpiece(y-z face) and two layers of atoms in the bottom of workpiece material(x-y plane). Atoms other than the boundary atoms are allowed to move freely during the process of simulation. For an MD simulation of different depths of cut using a similar cutting tool we know that larger the depth of cut greater the number of atoms to be removed hence more is the calculation time required for simulation. It also has been experimentally found that if the ratio of cutting depth to tip radius is greater than or equal to 0.09 , then the cutting is nanoscale cutting. In this study, the tip radius of the probe is diameter of the carbon atom. In the simulation, the cutting depths are $10 \AA, 15 \AA$ and $20 \AA$.The tool rake angles considered here are $0^{\circ}, 10^{\circ}, 20^{\circ}$ and $30^{\circ}$. Nanocutting is carried out for both Simple cubic (not real) and FCC (real) lattice structure of copper. The results for study of FCC crystal structure are described in following sections.

\subsection{Results and Discussion}

\subsubsection{Face Centered Cubic Lattice Structure}

Initially whole analysis above was conducted on an assumed simple cubic lattice structure of copper plate with a lattice constant of 3.61 . But in reality copper is found naturally in Face centered cubic lattice structure with same lattice constant, hence a similar analysis was done on Face centered cubic lattice of copper and the simulation results were displayed below. The results obtained for both lattice structures were similar except the values of cutting forces. Boundary conditions for this system are 4 layer of atoms along width and 2 layer of atoms along length are fixed (shown in red color)

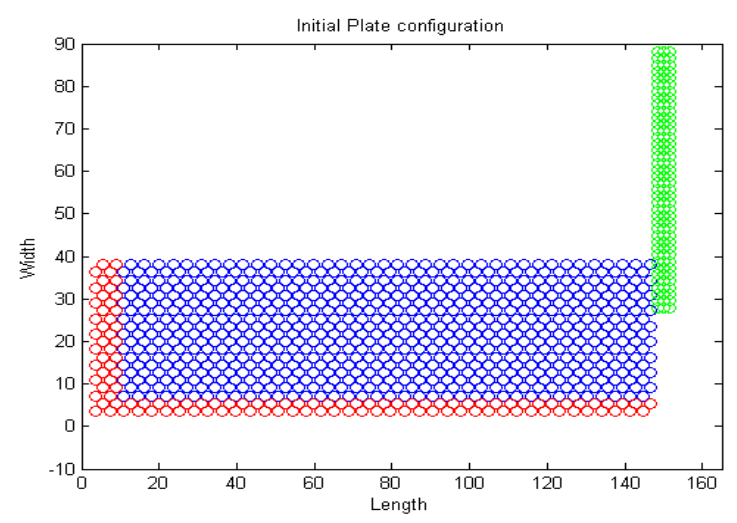

Fig 4 Simulation model for FCC lattice

\subsubsection{Effect of Depth of cut in FCC lattice}
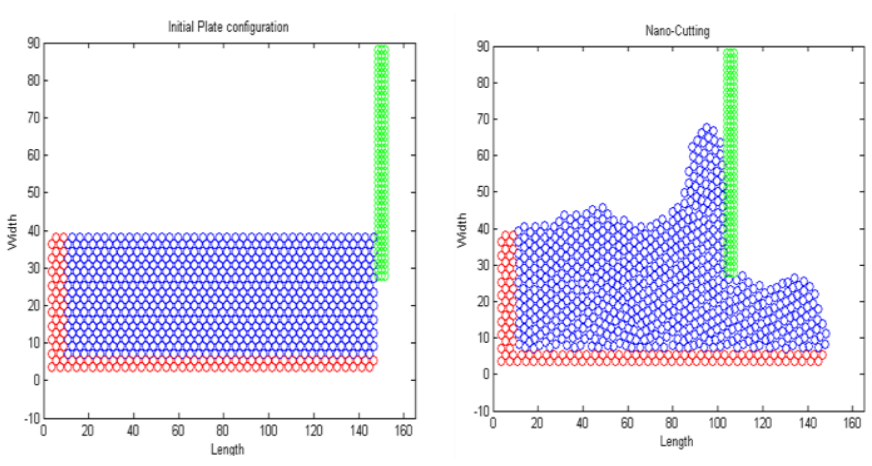

Fig 9 Simulation results for nanocutting after 4000th time step for FCC lattice of copper depth of cut: 10 Angstroms
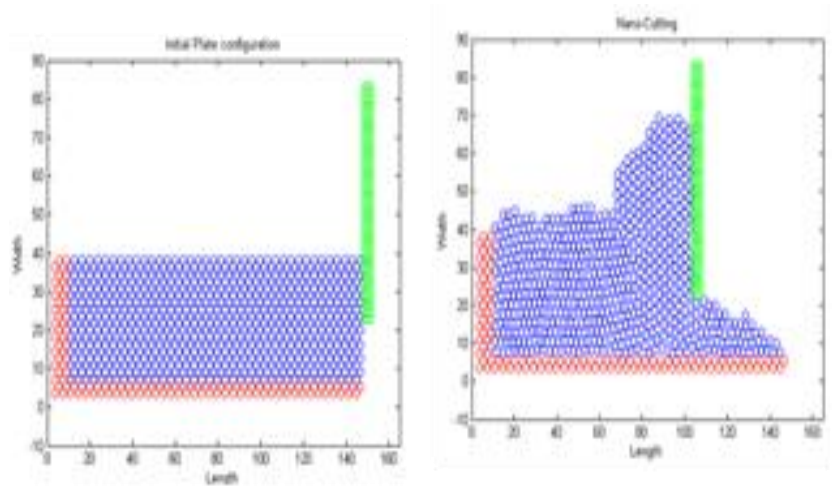

Fig 5 Simulation results for nanocutting after 4000th time step for FCC lattice of copper Tool rake angle: 10 degrees 

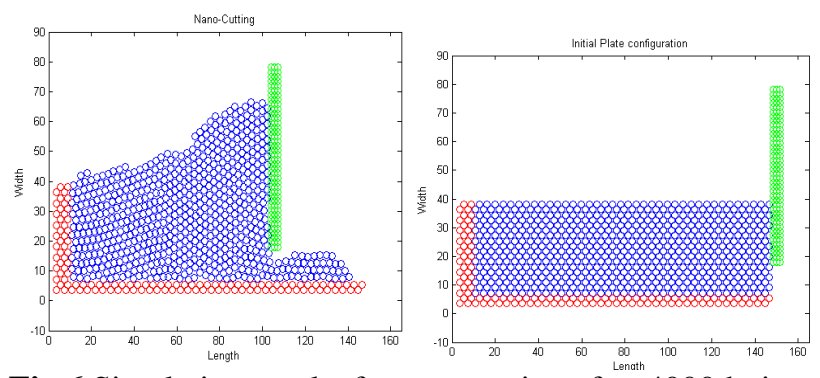

Fig 6 Simulation results for nanocutting after 4000th time step for FCC lattice of copper Tool rake angle: 10 degrees

\begin{tabular}{|l|l|}
\hline Configuration & Nanocutting \\
\hline Copper (Workpiece) & $144.3 \AA$ x $28.66 \AA$ \\
\hline Tool & $\begin{array}{l}\text { Rigid body Diamond } \\
4.62 \AA \times \text { x } 61.6 \AA\end{array}$ \\
\hline Tool rake angle & $10^{\circ}, 20^{\circ}$ and $30^{\circ}$ \\
\hline Tool clearance angle & $0^{\circ}$ \\
\hline Depth of cut $(\AA)$ & $10,15,20$ \\
\hline Cutting velocity $(\mathrm{m} / \mathrm{s})$ & 123 \\
\hline $\begin{array}{l}\text { Bulk Temperature } \\
(\mathrm{K})\end{array}$ & 298 \\
\hline
\end{tabular}

Simulations of nanocutting process of workpiece (here copper) with rigid tool (diamond) for different cutting conditions, using Molecular Dynamics simulation method were carried out. The following results were obtained:

- Nanocutting of copper using Diamond tool by Molecular Dynamics Simulations is found similar to the conventional machining process.

- Tangential Force is always greater than normal force.

- Cutting Forces depend on the depth of cut, tool rake angle and also lattice structure of the system under cutting.

- $\quad$ Both chip formation and irregularity of the machined surface depends on the depth of cut. Irregularity of machined surface and appearance of voids, dislocations in the cutting region increase with increase in depth of cut.

- $\quad$ Both tangential and normal cutting forces increase in magnitude with increase in depth of cut. Also their ratio increase but not linearly

- Chip formation and irregularity of machining surface is also influenced by tool rake angle. Higher the tool rake angle more the sleekness of the machined surface as atoms in cutting region are more close to each other.

- Cutting forces appear to be influenced by the variation of the tool rake angle, as well. As tool rake angle increases, cutting forces decrease considerably and the same happens with the ratio of cutting forces. Contrary to the depth of cut ratio of cutting forces decreases linearly in this case.

\section{FINDING MECHANICAL PROPERTIES}

Development and increased interest in studying the dynamics of fracture or breaking has lead the base for finding the mechanical properties of materials. Practically properties of materials can be found using tensile testing machine, UTM etc. But theoretically it is difficult to find as it involves different scales of length and time to analyze. Using Finite element method we can try to find out the properties at macroscopic level by reducing the grid size of analysis. Or we can use a combination of macroscopic and microscopic analysis known as Multi-scale modeling. Or we can entirely use microscopic analysis like Molecular dynamics to find the properties of the materials. As it is difficult to find the realistic potential at atomic scale we generally use pair-wise potential or many-body potential to approximate the potential energy of the simulation volume. Pair-wise potentials like Lennard-Jones (LJ) or Morse potential are often used as they approximate in a better way. However, when the results obtained using Morse or LJ potential are compared with experimental results we find that the pair-wise potential yield a brittle behavior while other yield ductile one. Hence we need to use many-body potential to obtain the ductile behavior. On the other hand, in simulations related to dynamic fracture we need to consider a large number of atoms for simulation, the increase in number of atoms will increase the computational space and time if we use many-body potential. Therefore pair-wise potentials are more preferred over many-body potential. But with the invention of supercomputers and advanced computational methods it is now possible in present world to simulate for a million atoms using manybody potential.

Also the elastic properties of copper using a realistic manyatom can now be obtained by ab initio type potential obtained from the effective-medium theory. Unlike the simulations by Zhou [11] (done practically at zero temperature) we have studied the system at room temperature. The objective of the following study is to observe the mechanism of tensile testing and the phenomenon using Molecular Dynamics Simulations and find the elastic constant of the material.

Virial stress is a also a widely used stress type at an atomic level. The virial stress is defined on the basis of virial theorem of Clausius (1870) developed for gas pressure. The virial stress is defined as:

$\sigma^{\text {virial }}(r)=\frac{1}{V} \sum_{i}\left[-m_{i} \dot{u}_{i} \boldsymbol{X} \dot{u}_{i}+\frac{1}{2} \sum_{j \neq i} r_{i j} \boldsymbol{X} f_{i j}\right]$

Here $i$ and $j$ are the atomic indices. The stress is calculated for whole simulation volume V. $m_{i}$ is the mass of atom $i$, $\dot{u}_{\tilde{i}}$ denotes the material time derivative of displacement relative to some reference frame and $\boldsymbol{X}$ represents the tensor product of two vectors. $\mathrm{f}_{\mathrm{ij}}$ is the interatomic force applied on atom $i$ by atom $j$.

However, using virial stress is not correct as it argues that the stress depends on both mass transfer in spatial region and force applied which is incorrect. Also the force applied here is the statistical average between the simulation volume and a non-deformable reference frame. Mechanical stress in general is the change in momentum of a particular mass element due to applied forces. Some of the other type of 
stresses defined at atomic level are BDT stress, Lutsko stress etc.BDT stress is put forward by Basinski, Duesbery, and Taylor (1971), and is based on a volumetric partition of the homogeneous deformed bulk by extending the virial stress to one atomic volume. BDT stress is defined as:

$$
\sigma_{a \beta}^{\prime}=\frac{1}{V_{s}}\left(-m_{t} v_{t}^{\alpha} v_{t}^{\beta}+\frac{1}{2} \sum_{f=i} r_{v}^{\alpha} f_{v}^{\beta}\right)
$$

Similarly Lutsko stress is defined as:

$$
\sigma_{a \beta}^{\prime}=\frac{1}{V_{i}}\left(-m_{t} v_{t}^{\alpha} v_{t}^{\beta}+\frac{1}{2} \sum_{j \neq i} r_{i}^{\alpha} f_{v}^{\beta}\right)
$$

In our study we have used BDT stress for stress calculation using Molecular Dynamics Simulation. Only the initial model without taking into consideration of factors like voids, surface effects etc... is used for tensile testing.

\subsection{Results and Discussions}

\subsubsection{Model Specifications}

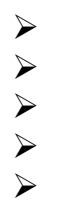

$\begin{array}{lc}\text { Material } & - \text { Copper } \\ \text { Model Type } & -3 \mathrm{D} \\ \text { Dimensions } & -27 \AA \text { x } 56 \AA \text { x } 27 \AA \\ \text { Constant strain rate } & -100 \mathrm{~m} / \mathrm{s} \\ \text { Stress } & -\mathrm{BDT}\end{array}$

\subsubsection{Young's Modulus (Elastic region)}

Experimental: 120GPa

By MD Simulation: $919 \mathrm{GPa}$

Some of the possible reasons for above results may be

- The Concept of stress at an atomic level

- Application of Constant strain rate

- Incorrect Application of Periodic Boundary Conditions(PBCs)

The results obtained after running the simulation for around 2000 time steps (with time step $1 \mathrm{fs}$ ) are displayed below:

\section{CONCLUSION}

The pattern of Tensile testing was found similar to the conventional one except the boundaries weren't smooth surfaces. By choosing a better atomic stress, by tuning the method of calculating elastic modulus and applying a time dependent strain rate the results may improve. Application of constant strain rate produces incorrect values of Young's Modulus (Elastic).
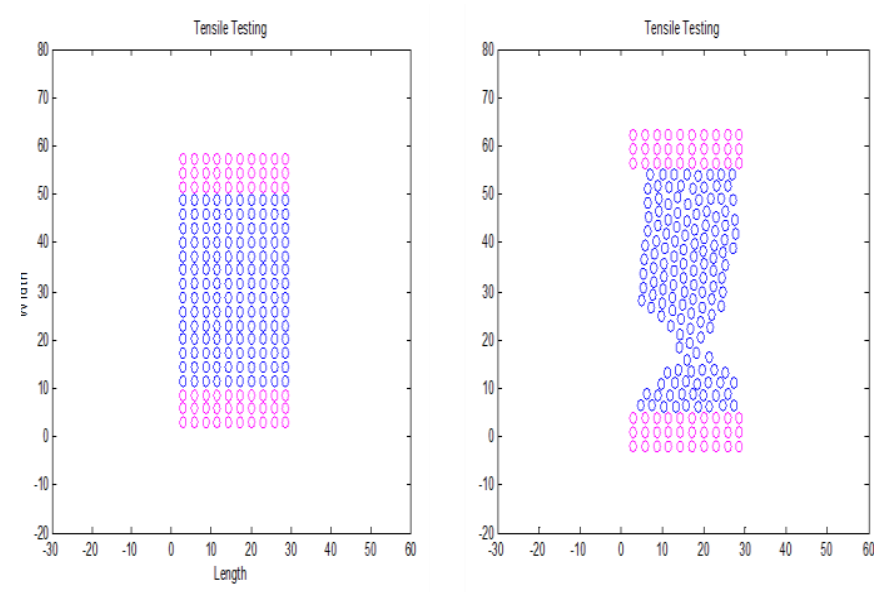

Fig 12: Tensile Test Patteren

\section{REFRENCES}

[1] D Keffer, The Working person's guide to Molecular Dynamics Simulation (2001)

[2] J M Haile, Molecular Dynamics Simulation: Elementary Methods

[3] Wei Cai, An overview of Molecular Dynamics

[4] K.S. Sharma, C.M.Kachhava, Application of Morse Potential to Metals in Molecular- Metallic Framework (2008)

[5] Jen Ching Huang, The study on Nano-cutting by a rigid body tool and elastic body tool using Molecular Dynamics Simulation (2013)

[6] Experimental Values and Parametersvhttp://www.knowledgedoor.com/2/eleme nts handbook/

[7] Shengping shen, S.N.Atluri, Atomic-level stress calculation and Continuum- Molecular System Equivalence (2004)

[8] N. Chandrasekaran, A. Noori Khajavi, L. M. Raff and R. Komanduri, "A New Method for MD Simulation of Nanometric Cutting," Phil. Mag. B., vol. 77, no. 1, pp. 7-26, 1998.

[9] R. Komanduri, N. Chandrasekaran and L. M. Raff, "Some aspects of machining with negative-rake tools simulating grinding a molecular dynamics simulation approach," Philos. Mag. B, vol. 79, no. 7, pp. 955-968, 1999

[10] Huang, Jen Ching, and Fu Jen Cheng. "The Nanocutting by Rigid/Elastic Tools with Nose Radius at the Gas Environment Using Molecular Dynamics Simulations", Applied Mechanics and Materials, 2014.

[11] Zhou S. J., Beazley D. M., Lomdahl P. S. and Holian B. L., Phys. Rev. Lett., 78 (1997) 479 